\title{
DP Capability Analysis for HYSY981
}

\author{
Dongshi Wang ${ }^{1, a}$, Bo Yan, b ${ }^{1,}$ Fengxi Gai, c, Wenhua $\mathrm{Li}^{2, \mathrm{~d}}$ and Gang Wang ${ }^{2, \mathrm{e}}$ \\ ${ }^{1}$ China Oilfield Services LTD, Sanhe 065200, China; \\ ${ }^{2}$ School of Marine Engineering, Dalian Maritime University, Dalian 116000, China. \\ awangdsh4@cosl.com.cn, byanbo2@cosl.com.cn, cfxgai@sina.com, dliwenhua992@ailyun.com, \\ ewanggang@dlmu.edu.cn
}

Keywords: HYSY981, dynamic positioning capability, analysis.

\begin{abstract}
Hai Yang Shi You 981 (HYSY981) is a 6th generation deep water Semi-submersible rig and the first DP3 drilling rig in China. To ensure safety operation of the rig, dynamic positioning capability analysis was performed on HYSY981. Firstly, the model of marine environment including wind, wave and current was established to simulate real marine environment loads. Then different failure modes were designed, such as loss of one or more thrusters and loss of one or more switchboards. The designed failure modes were applied to the established model of dynamic positioning system of HYSY981. Then under marine conditions and designed different failure modes as above, some dynamic positioning capability simulation analyses for HYSY981 were done. This simulation analysis results showed that the DP station keeping performance is excellent. Eventually, some practical failure modes such as drive off due to two DGPS drift or conditions such as soliton disturbance in the South China Sea in the operation of HYSY 981 were given, the reliable DP capability of HYSY981 was verified, at the same time the DP capability simulation analyses were proved right.
\end{abstract}

\section{Introduction}

The Hai Yang Shi You 981 (HYSY981) is a $6^{\text {th }}$ generation semi-submersible deep water drilling unit registered in the People's Republic of China as shown in Fig.1. It was equipped DP-3 system, Kongsberg's production, and the DP system was upgraded to adapt to the South China Sea area. Dynamic positioning system is a computer-based automatic positioning system for floating vessels, the thrusters are managed by a control system (DP) to keep the vessel on a constant heading and in a constant position, compensating for forces from wind, current and wave action [1]. How to analyze and evaluate the DP capability is practical and significant. DP capability plot is a powerful tool to do that [2]. Therefore, research on DP capability plot can objectively reflect the actual performance and capability of HYSY981.Principal molded dimensions and design operating conditions are given by Tables 1 and 2 below.

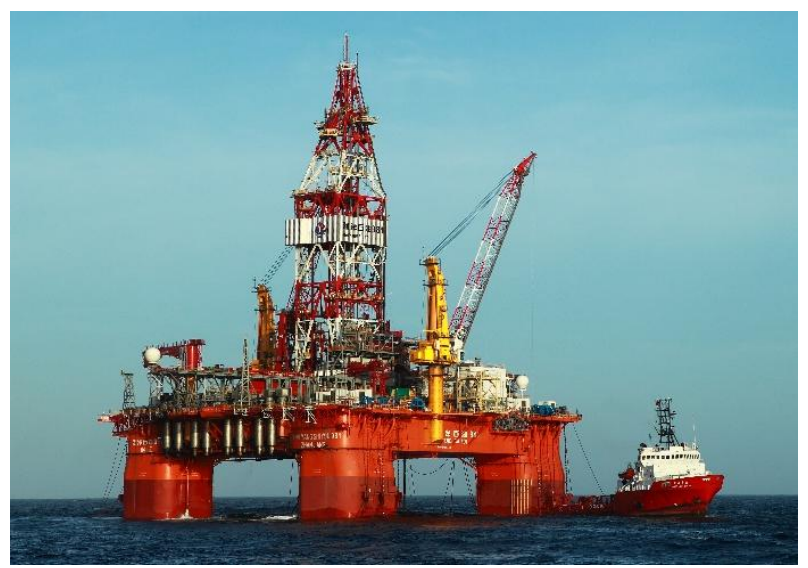

Fig. 1 Hai Yang Shi You 981 
Table 1 Principal Dimensions

\section{Pontoons}

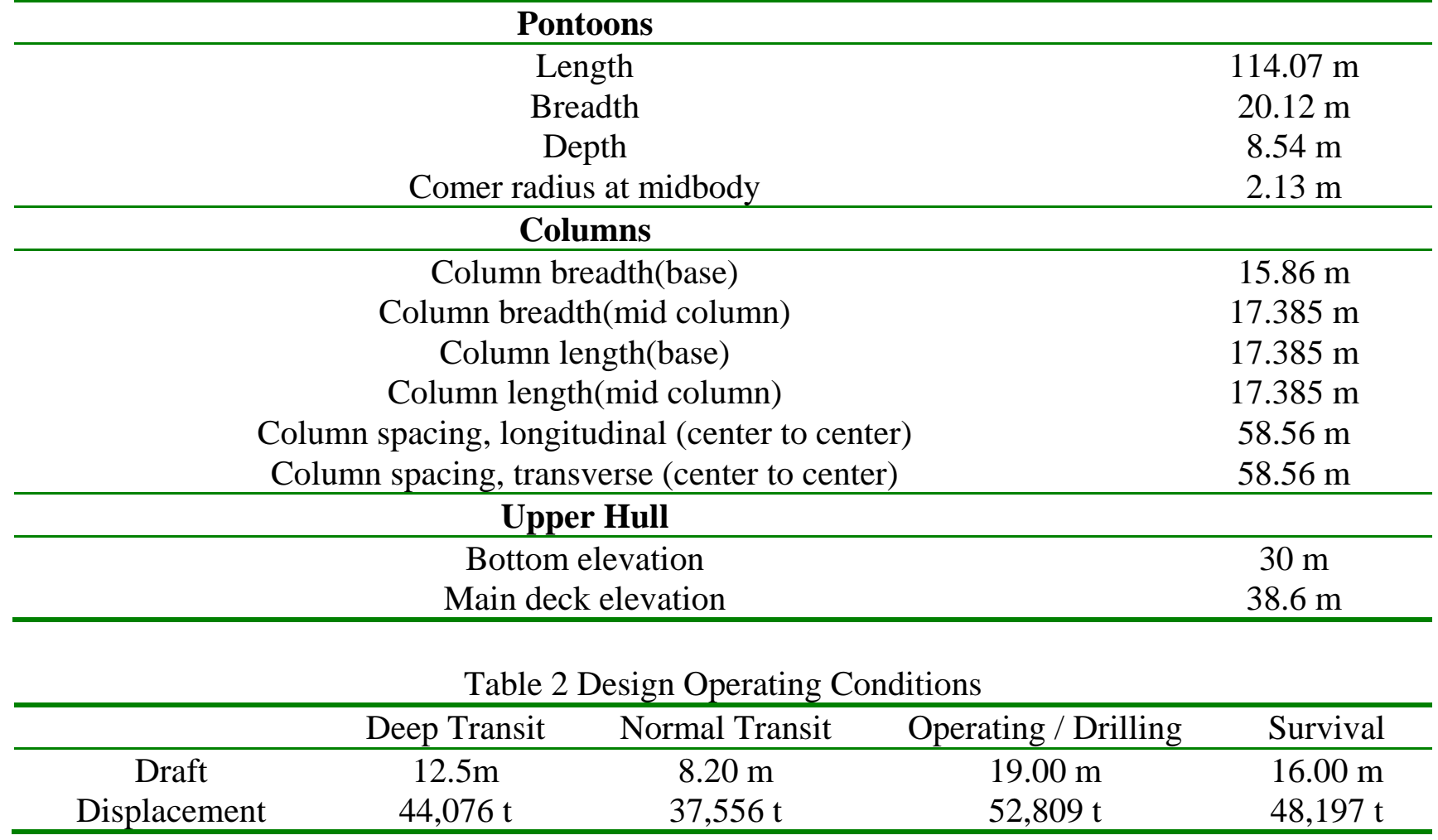

\section{Coordinate System}

The coordinate system used is the orthogonal right-handed system shown in Fig. 2 with the positive $z$-axis pointing downwards. The origin of the coordinate system can be offset a longitudinal distance $\mathrm{x}_{0}$ from $\mathrm{Lpp} / 2$. The directions of the wind, waves and current are defined by means of coming-from directions and are considered positive when turning clockwise, e.g. a wind direction equal to 0 degrees exerts a negative longitudinal force on the vessel. Unless otherwise stated, the directions of the wind, waves and current are coincident in the analyses.

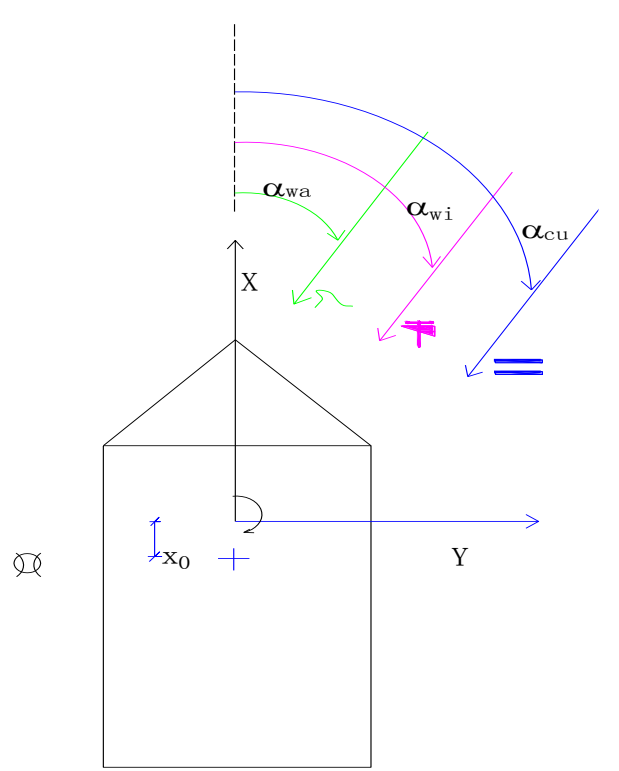

Fig. 2 Coordinate system and sign conventions 


\section{Thruster}

\subsection{Thruster Performance.}

HYSY 981 is equipped with 8 Wartsila azimuthing thrusters for DP propulsion (see Fig.3). These thrusters are $3600 \mathrm{~mm}$ in diameter and are in a kort nozzle. They have a maximum power input of $4600 \mathrm{~kW}$ at $600 \mathrm{rpm}$ from the motor and deliver $4462 \mathrm{~kW}$ at $181 \mathrm{rpm}$ through an L-drive. Combined power loss from the generator, through the distribution system, converters, motor and gear boxes is typically in the region of $6 \sim 8 \%$. It will be taken as $7 \%$ for intact conditions and $8 \%$ for in damaged conditions in order to be conservative. Open water thruster performance curves are showed in Fig.4.

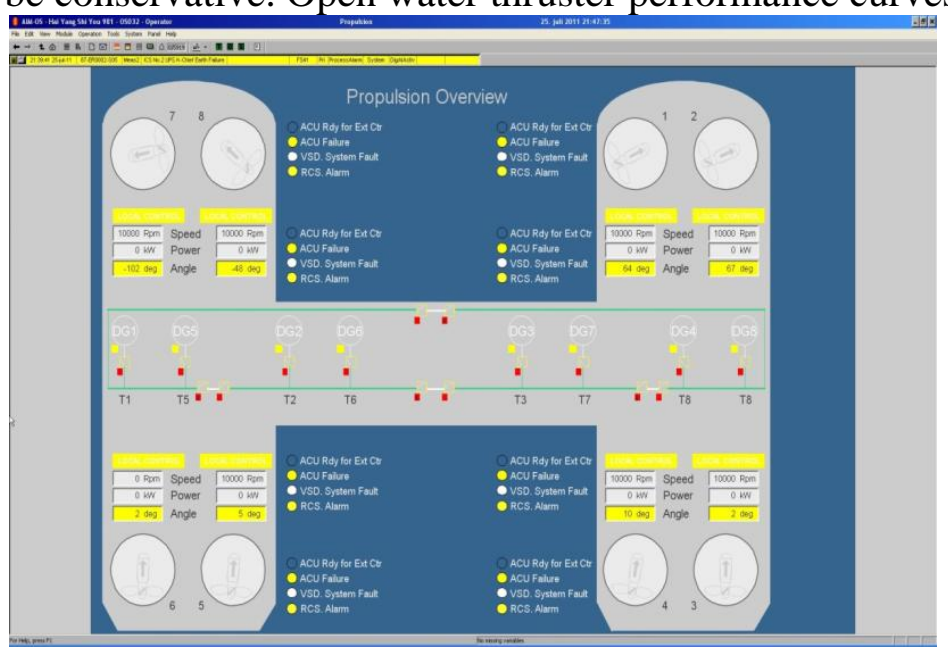

Fig. 3 Thruster layout

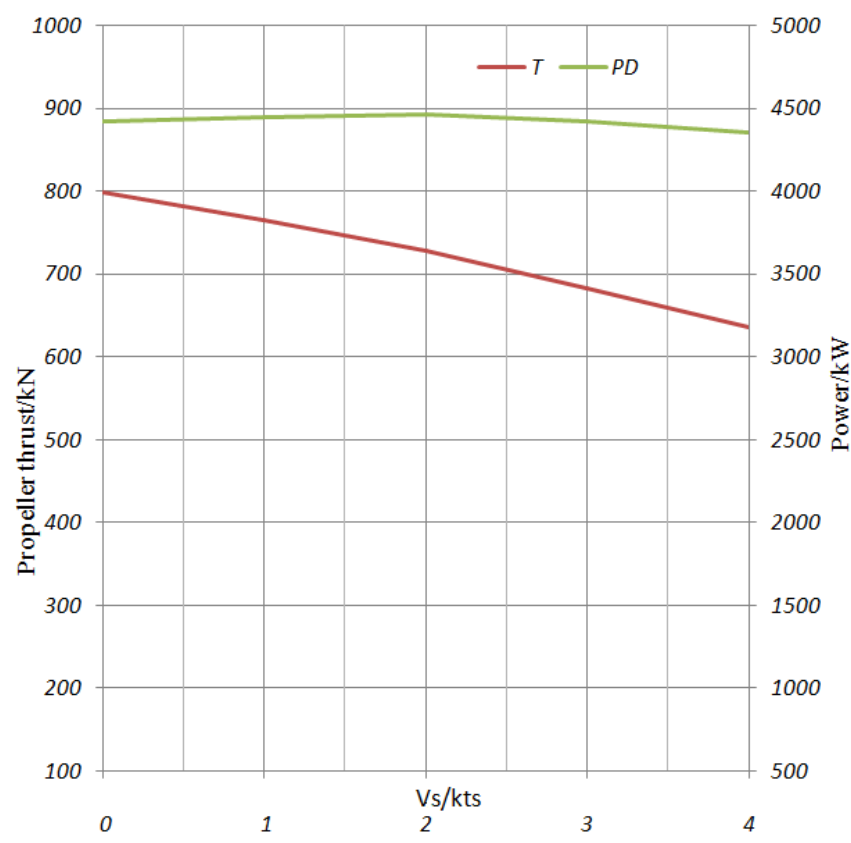

Fig. 4 Open water thruster performance curves-Propeller thrust and power

\subsection{Thruster-thruster Interactions.}

Thruster-thruster interactions occur when the wake from one thruster impinges on the wake from another thruster. Thruster-hull interactions arise from the Coanda effect and impingement of thruster wakes on the other pontoon. Both of these effects are included in the thruster interaction curves (see Table3). The forbidden zones are regions of high efficiency loss due to thruster-thruster impingement effects. For headings where one thruster was directly in the wash of another upstream, both thrusters were each taken to be rotated 7.5 degrees to opposite sides of the environment axis to minimize thruster-thruster interaction. A $180^{\circ}$ correction was made to ensure the thruster heading opposed the environment. 
Table 3 Thruster Efficiency versus Thrust Heading

\begin{tabular}{|c|c|c|c|c|c|c|}
\hline \multirow[b]{2}{*}{ Azimuth } & \multicolumn{4}{|c|}{ Thruster Efficiency } & \multicolumn{2}{|c|}{ Range } \\
\hline & 1.2 & 3.4 & 7.8 & 5.6 & $\min$ & $\max$ \\
\hline 0 & 0.82 & 0.82 & 0.93 & 0.93 & 82 & 93 \\
\hline 15 & 0.9 & 0.89 & 0.94 & 0.92 & 89 & 94 \\
\hline 30 & 0.84 & 0.92 & 0.95 & 0.91 & 84 & 95 \\
\hline 45 & 0.78 & 0.93 & 0.96 & 0.9 & 78 & 96 \\
\hline 60 & 0.73 & 0.95 & 0.97 & 0.89 & 73 & 97 \\
\hline 75 & 0.67 & 0.83 & 0.8 & 0.84 & 67 & 84 \\
\hline 90 & 0.61 & 0.66 & 0.61 & 0.66 & 61 & 66 \\
\hline 105 & 0.8 & 0.84 & 0.67 & 0.83 & 67 & 84 \\
\hline 120 & 0.97 & 0.89 & 0.73 & 0.95 & 73 & 97 \\
\hline 135 & 0.96 & 0.9 & 0.78 & 0.93 & 78 & 96 \\
\hline 150 & 0.95 & 0.91 & 0.84 & 0.92 & 84 & 95 \\
\hline 165 & 0.94 & 0.92 & 0.9 & 0.89 & 89 & 94 \\
\hline 180 & 0.93 & 0.93 & 0.82 & 0.82 & 82 & 93 \\
\hline 195 & 0.92 & 0.94 & 0.89 & 0.9 & 89 & 94 \\
\hline 210 & 0.91 & 0.95 & 0.92 & 0.84 & 84 & 95 \\
\hline 225 & 0.9 & 0.96 & 0.93 & 0.78 & 78 & 96 \\
\hline 240 & 0.89 & 0.97 & 0.95 & 0.73 & 73 & 97 \\
\hline 255 & 0.84 & 0.8 & 0.83 & 0.67 & 67 & 84 \\
\hline 270 & 0.86 & 0.61 & 0.66 & 0.61 & 61 & 66 \\
\hline 285 & 0.83 & 0.67 & 0.84 & 0.8 & 67 & 84 \\
\hline 300 & 0.95 & 0.73 & 0.89 & 0.97 & 73 & 97 \\
\hline 315 & 0.93 & 0.78 & 0.9 & 0.96 & 78 & 96 \\
\hline 330 & 0.92 & 0.84 & 0.91 & 0.95 & 84 & 95 \\
\hline 345 & 0.89 & 0.9 & 0.92 & 0.94 & 89 & 94 \\
\hline
\end{tabular}

\subsection{Thruster Allocation.}

The thruster allocation algorithms were defined using Lagrange multipliers to minimize a cost function. The objective of the optimization problem is to hold station while minimizing power. It is assumed that power Thrust ${ }^{2}$. Power is minimized subject to the constraints that the rig maintain static equilibrium as defined by the following equations:

$$
\begin{aligned}
& \sum_{i=1}^{8} T_{x i}=X_{R E Q} . \\
& \sum_{i=1}^{8} T_{y i}=Y_{R E Q} . \\
& \sum_{i=1}^{8}\left(d_{x i} T_{y i}-d_{y i} T_{x i}\right)=M_{R E Q} .
\end{aligned}
$$

where $X_{R E Q}, Y_{R E Q}, M_{R E Q}$ are the total environmental loads in the $x, y$ and yaw senses. $T_{x i}$ and $T_{y i}$ are the $x, y$ components of the thrust vector from thruster $i, d_{x i}, d_{y i}$ are the $x, y$ coordinated of thruster $i$ from the center.

This leads to the following cost function in which the three Lagangre multipliers are applied to the constraint equations:

$$
\begin{aligned}
\cos t= & \sum_{i=1}^{8} T_{x i}^{2}+\sum_{i=1}^{8} T_{y i}^{2}+\lambda_{1}\left(\sum_{i=1}^{8} T_{x i}^{2}-X_{R E Q}\right)+ \\
& \lambda_{2}\left(\sum_{i=1}^{8} T_{y i}^{2}-Y_{R E Q}\right)+\lambda_{3}\left(\sum_{i=1}^{8}\left(d_{x i} T_{y i}-d_{y i} T_{x i}\right)\right.
\end{aligned} .
$$

This cost is minimized by taking the partial derivative of the cost function with respect to each variable. Noting that the minima will occur when the first derivative is zero, we get a set of 
independent linear equations which can be solved by matrix inversion to yield expressions for the thruster components.

For the intact condition, the following equations were derived:

$$
\begin{aligned}
& T_{x 1}=0.125 X_{R E Q}-0.0014312 M_{R E Q} \\
& T_{x 2}=0.125 X_{R E Q}-0.0009739 M_{R E Q} . \\
& \vdots \\
& T_{y 8}=0.125 Y_{R E Q}-0.0019083 M_{R E Q}
\end{aligned}
$$

\section{Environment Loads}

\subsection{Wind loads.}

Wind loads were calculated using:

$\vec{F}=W_{c} \cdot V^{2}$.

where $F=$ wind load in tonnes; $W_{c}=$ wind coefficient $\left(\mathrm{te} /(\mathrm{m} / \mathrm{s})^{\wedge} 2\right)$; and $V=$ wind speed $(\mathrm{m} / \mathrm{s})$. Wind coefficient derived from wind tunnel test, see Fig.5, according to Ref. [3].

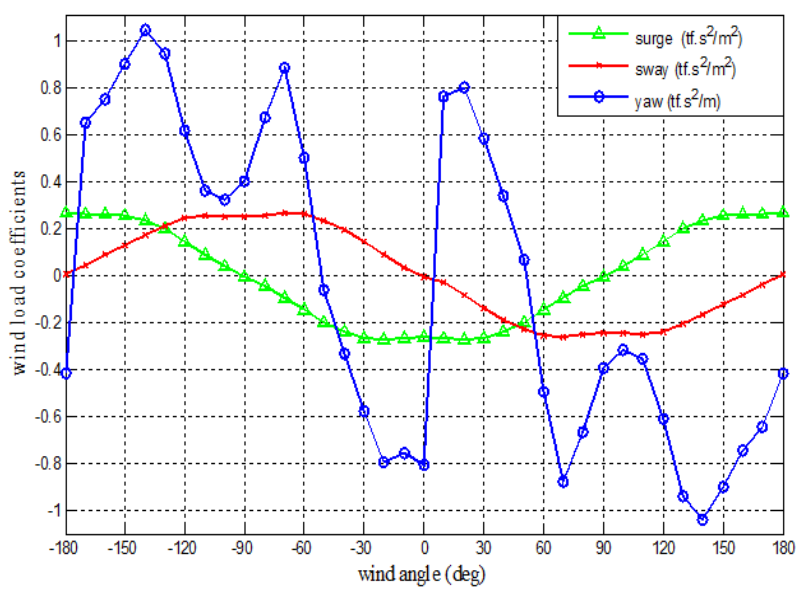

Fig. 5 Wind load coefficients

\subsection{Wave loads.}

The wave drift loads were calculated using:

$\vec{F}=W_{d} \cdot H_{s}{ }^{2}$.

where $F=$ wave load in tonnes; $W_{d}=$ wave drift coefficient (tonnes $\left./ \mathrm{m}^{\wedge} 2\right) ; H_{s}=$ significant wave height $(\mathrm{m})$. The wave drift coefficients were derived by processing the results from the hydrodynamic analysis (see Fig.6, 7 and 8).

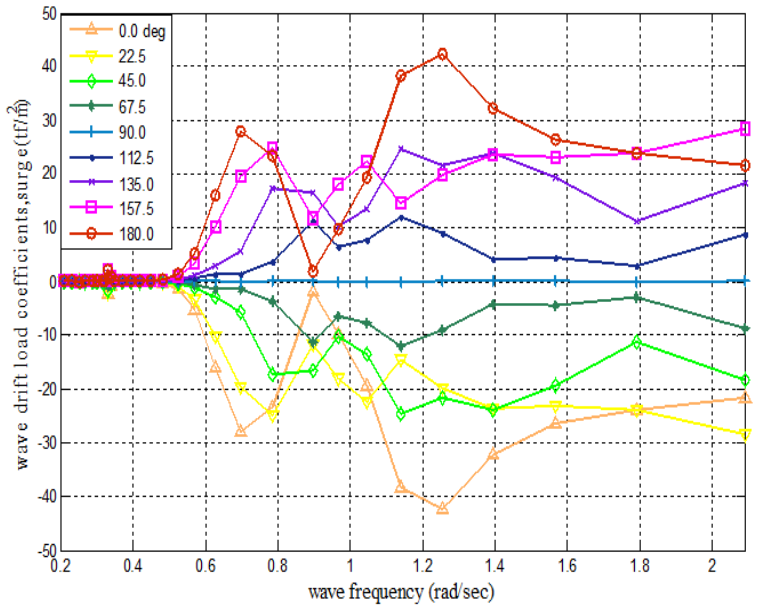

Fig. 6 Wave-drift load coefficients for surge 


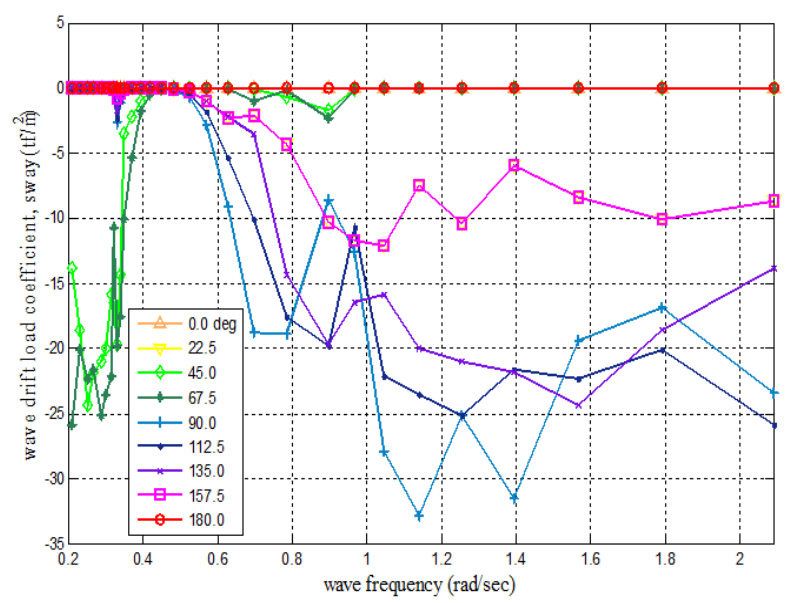

Fig. 7 Wave-drift load coefficients for sway

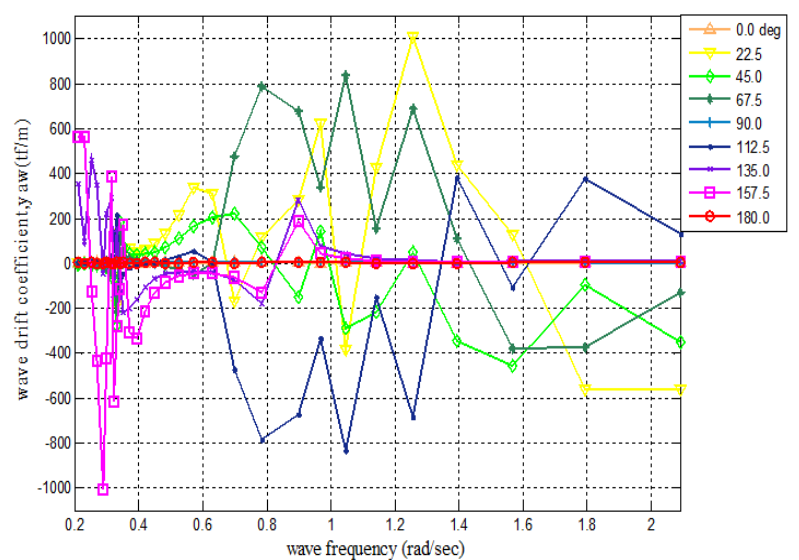

Fig. 8 Wave-drift load coefficients for yaw

\subsection{Current loads.}

Current loads were calculated using:

$\vec{F}=C_{c} \cdot V^{2}$.

where $F=$ current load in tonnes;

$C_{c}=$ current coefficient $\left(\mathrm{te} /(\mathrm{m} / \mathrm{s})^{\wedge} 2\right)$ in three directions;

$V=$ current speed $(\mathrm{m} / \mathrm{s})$. Current load coefficients are showed in Fig.9.

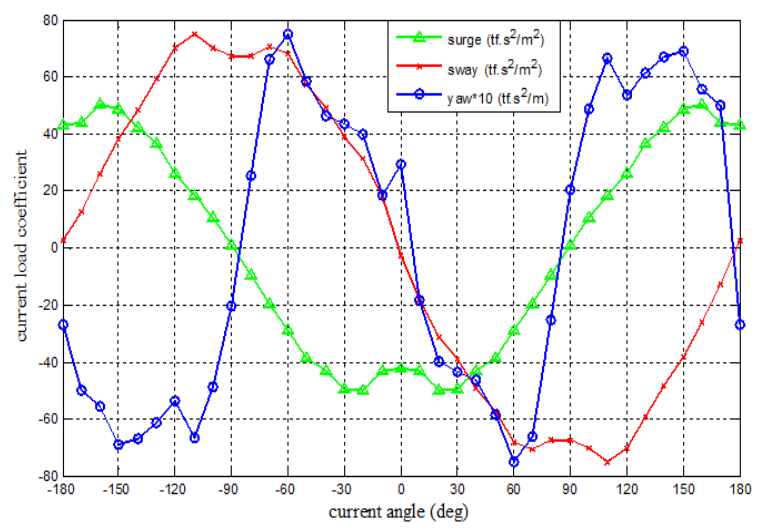

Fig. 9 Current load coefficients

\section{DP CAPABILITY}

DP capability defines a DP vessel's station-keeping ability under given environmental and operational conditions [4]. DP capability analyses are generally used to establish the maximum weather conditions in which a DP vessel can maintain its position and heading for a proposed thruster 
configuration. The environmental forces and moments are increased until they are balanced by the maximum available thrust offered by the thruster configuration. So the environment loads and thrust must meet the equality:

$$
\begin{aligned}
& \sum_{i=1}^{8} T_{x i}=X_{R E Q} . \\
& \sum_{i=1}^{8} T_{y i}=Y_{R E Q} . \\
& \sum_{i=1}^{8}\left(d_{x i} T_{y i}-d_{y i} T_{x i}\right)=M_{R E Q} .
\end{aligned}
$$

where $X_{R E Q}, Y_{R E Q}, M_{R E Q}$ are the total environmental loads in the $x, y$ and yaw senses. $T_{x i}$ and $T_{y i}$ are the $x, y$ components of the thrust vector from thruster $i, d_{x i}, d_{y i}$ are the $x, y$ coordinated of thruster $i$ from the center.

By allowing the environmental components to rotate in steps around the vessel, the results of a DP capability analysis can be presented by means of a limiting mean wind speed for a series number of wind angles of attack. The resulting polar plot is often referred to as a DP capability envelope.

\section{SIMULATION UNDER GIVEN CONDITIONS}

\subsection{Simulation.}

The simulation case definitions are given [5] in Table 4. T1 denotes thruster number 1 and so on. Based on the above thrust system parameters and environmental load parameters, Results were calculated by the designed program at intervals of 10 degrees wind direction. Results are showed as Fig.10 Fig.15.

Table 4 Simulation case definitions

\begin{tabular}{|c|c|c|c|}
\hline Case No. & $\begin{array}{c}\text { Current speed } \\
\text { kts }\end{array}$ & Thrusters active & Case description \\
\hline 1 & 1.5 & T1-T8 & Environmental Regularity Numbers [6] \\
\hline 2 & 1.5 & T1-T3, T5-T8 & Loss of one thruster \\
\hline 3 & 1.5 & T1, T3-T8 & Loss of one thruster \\
\hline 4 & 2.0 & T2-T4, T6-T8 & Loss of switchboard MV1 \\
\hline 5 & 2.0 & T1, T3-T5, T7-T8 & Loss of switchboard MV2 \\
\hline 6 & 2.0 & T3-T8 & Loss of switchboard forward corner \\
\hline
\end{tabular}

\subsection{Results.}

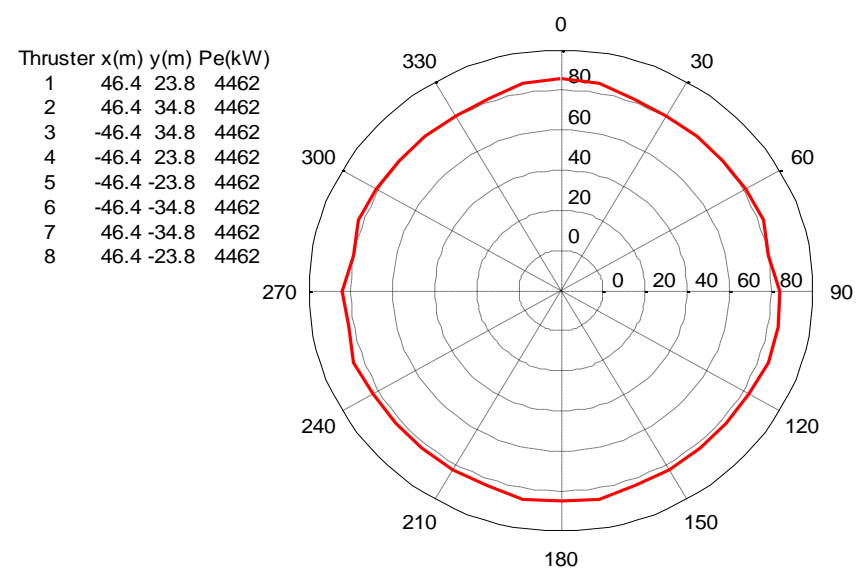

Fig. 10 Case1: all thruster enabled 


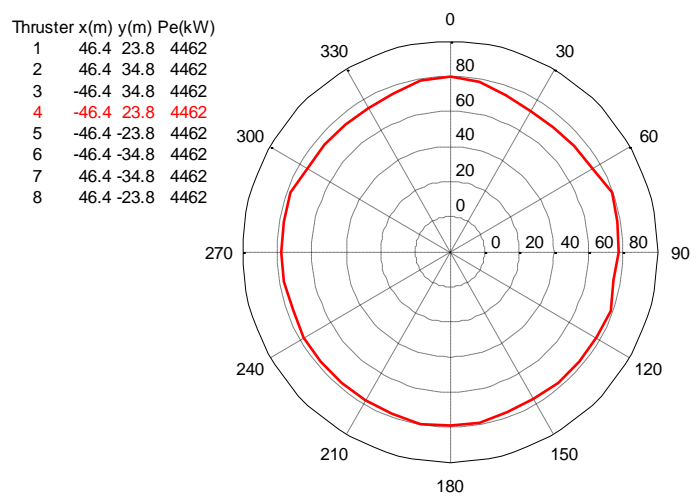

Fig. 11 Case2: loss of least significant thruster-T4

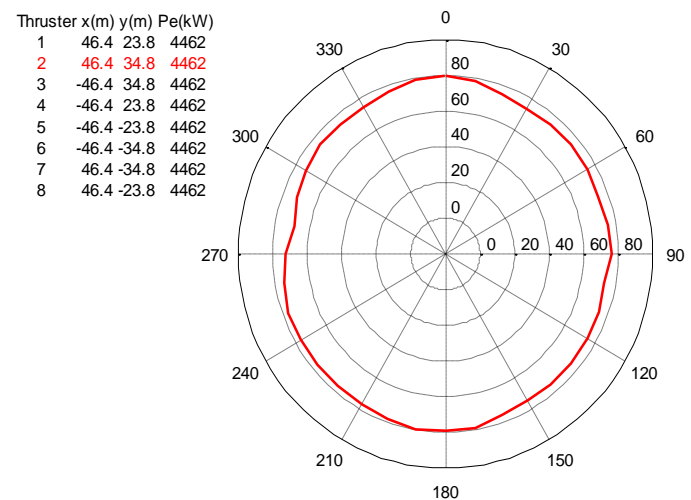

Fig. 12 Case3: loss of most significant thruster-T2

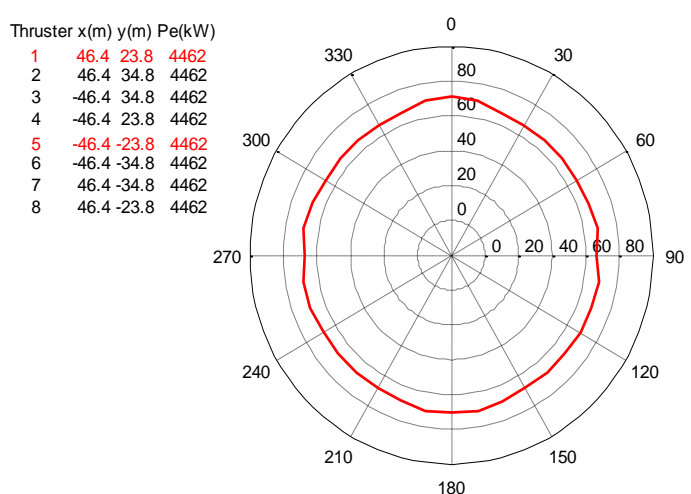

Fig. 13 Case4: loss of switchboard MV1-T1,T5

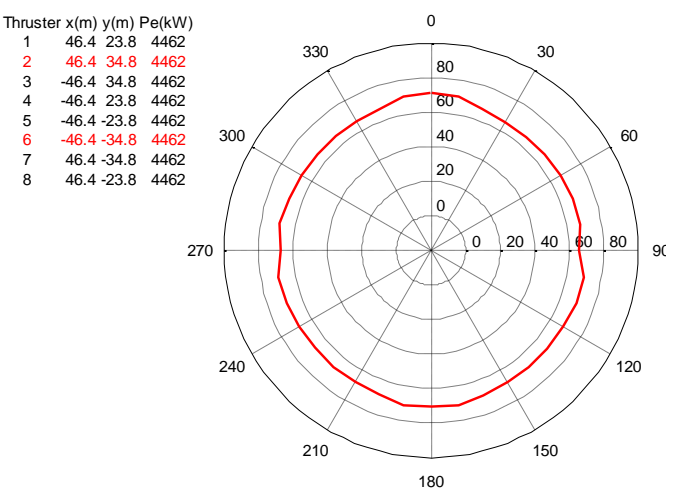

Fig. 14 Case5: loss of switchboard MV2-T2,T6 


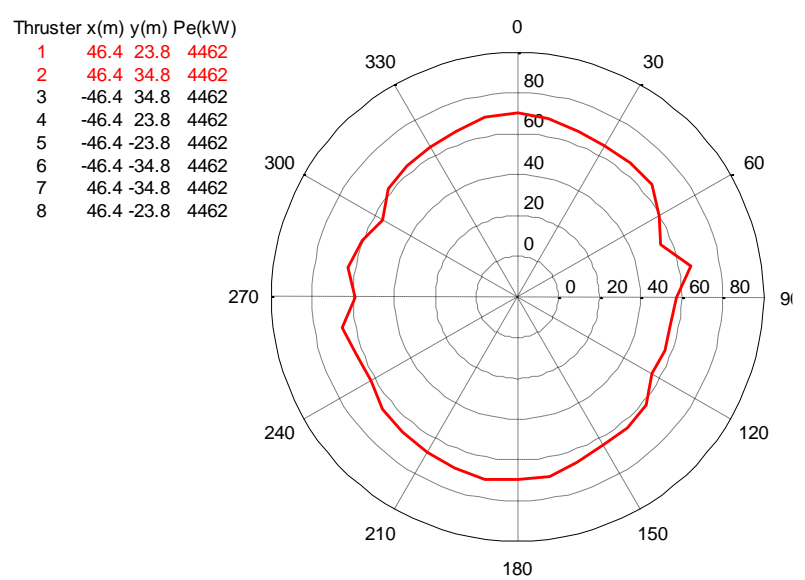

Fig. 15 Case6: loss of switchboard forward cormer-T1, T2

In general, the simulations indicate good DP station keeping behavior. The different simulation results show that:

(1) When all thrusters are operating in normal (case 1), the platform can resist larger environmental loads in $0^{\circ} \sim 360^{\circ}$ direction, especially in the longitudinal and transverse directions. Therefore, the integrated performance of dynamic positioning system of HYSY 981 meets the DP3 design requirements.

(2) When a single thruster failure occurs (case 2 and case 3), the DP capability will be weakened to a certain extent. The environmental loads can be resisted by the platform will decrease. Especially the failure mode of the outer ring thruster (T2, T3, T6 and T7) will have a greater impact.

(3) When there are two thrusters failures at the same time, the DP capability is further weakened. Compared with the two adjacent thrusters failures in the same floating body (case 6), the DP capability is better when the two diagonal thrusters are out (case 4 and case 5). The shape of DP capability plot is similar to the normal. While, if the two adjacent thrusters in the same floating body are failed, the DP capability will have a serious deterioration, and it is more obvious in some particular directions.

\section{Safety Analysis of Hysy981 Dp System}

Practice is the sole criterion for testing truth. Many practical failure modes occur such as two DGPS drift or soliton disturbance in South China Sea in the operation of HYSY 981. These failure models and environment disturbance also verified the DP capability.

\subsection{Failure Mode of DGPS.}

HYSY981 is equipped with DGPS's DPS 132 and two DPS 232, used as GPS1, GPS2 and GPS3.GPS1 and GPS2 occurred reference prediction error and reference high offset failure modes, the data from them is lost, but the GPS3 as the redundant equipment is enabled, so 5m drived off due to two DGPS drift, within the allow able range. Therefore, the DP3 system equipped in HYSY 981 has a good DP capability.

\subsection{Soliton Disturbance in the South China Sea.}

The drilling unit encountered the soliton many times when operating in South China Sea. The characteristic of it are that: current speed is strong, can reach 3.7 knots; duration is short, about 20 minutes; velocity and direction changed at the same time; cyclicity and continuity; easy to use visual or radar observation when calm.

The effect of soliton on DP are that when the soliton arrives, there action time of DP system has a certain delay and when the soliton flows out of the dynamic positioning system, the reaction time is lagged; the platform position will drift.

According to experience, the treatment guidance when encountering soliton are that: (1) All diesel generators are set to "auto start"; (2) Dynamic Positioning Operator (DPO) should use various means including visual and radar to pre-found soliton and its intensity; (3) DPO should start all the thrusters and generators in advance to cope with the incoming soliton; (4) DPO should adjust the heading of 
the platform to be consistent with the direction of soliton; (5) Correctly using the "Quick Mode $\mathrm{t}$ " function of DP system according to the actual situation; (6) Notify the relevant departments to take necessary follow-up, according to the soliton strength to decide whether to enter a state of emergency, and suspending tug berthing, helicopter landing operation; (7) Return to normal, lift the warning status.

Under the action of most strong soliton, the largest drift is $4.9 \mathrm{~m}$ within the allowable range according to the records on interface of the K-Pos DPM-32. Therefore, the reliable DP capacity can ensure the safety of production operation.

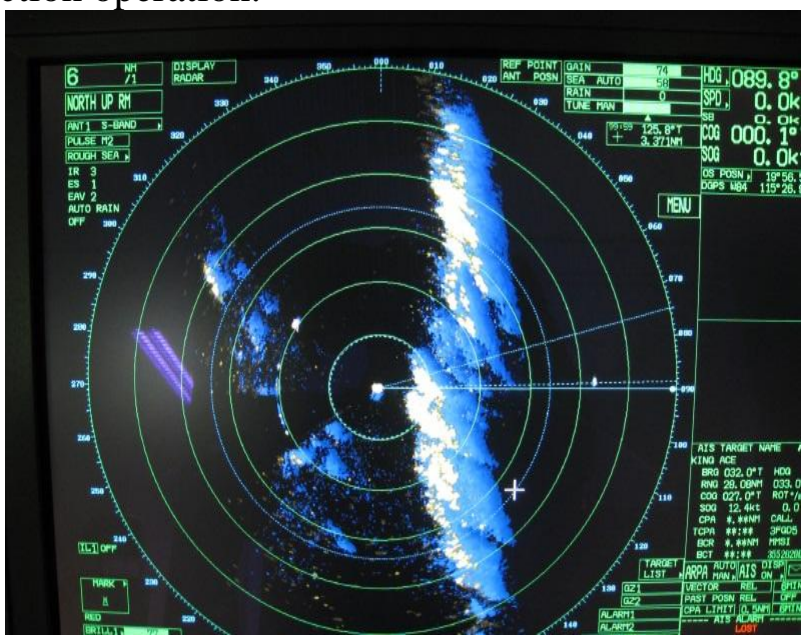

Fig. 16 Radar image about soliton

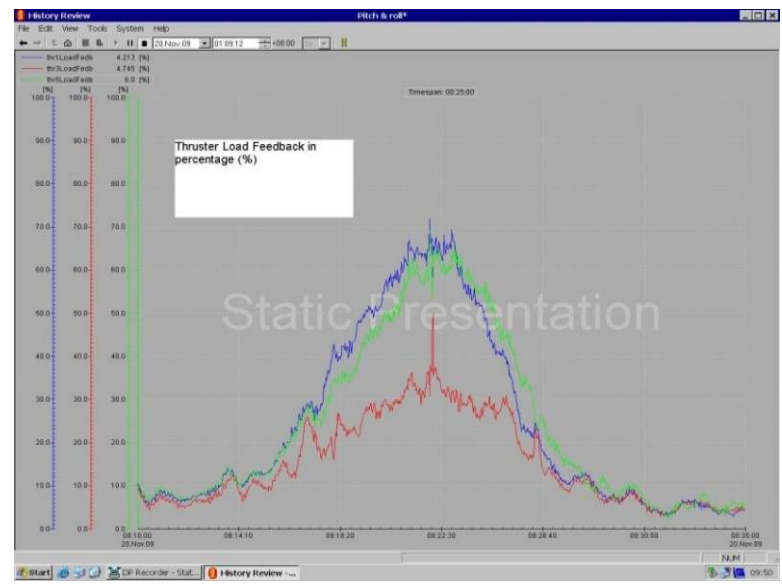

Fig. 17 Soliton data analysis

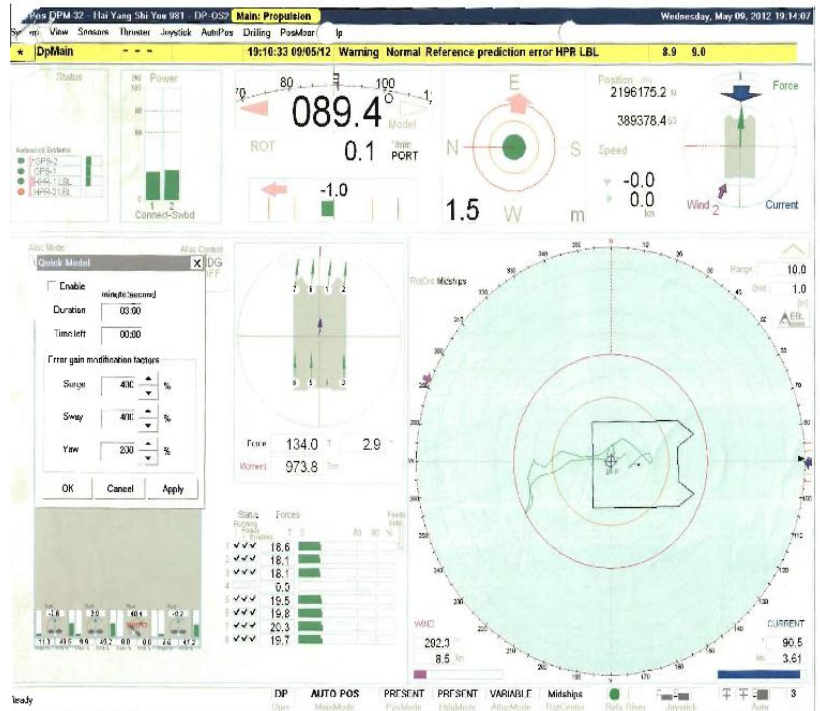

Fig. 18 Interface of the K-Pos DPM-32 


\section{Conclusion}

In a word, theoretical calculation and simulation were done to verify DP station keeping performance of HYSY981 is good. The actual failure modes and environment disturbance such as soliton acted on the drilling unit had proved the reliable DP capability.

\section{Acknowledgements}

This paper is sponsored by the Fundamental Research Funds for the Central Universities 3132016344, 3132016356, 3132016345) and Natural Science Foundation of Liaoning Province (2015020132).

\section{References}

[1]. International Maritime Organization. Guidelines for vessels with dynamic positioning systems. IMO MSC Circular 645, 1994.

[2]. Björn von Ubisch. Station Keeping Criteria for Dynamically Positioned Vesse. Dynamic Positioning Conference. Houston, September 28-30, 2004, p. 1-6.

[3]. Hai Yang Shi You 981. Stationkeeping Analysis for DDU Semisubmersible Drilling Rig, 2008.

[4]. IMCA M 140 Rev.1. The International Marine Contractors Association Specification for DP capability plots, 2000

[5]. KONGSBERG. DP Capability Analysis 19 m Draught CNOOC Hai Yang Shi You 981, 2012.

[6]. DNV•GL. Rules for classification of ships. Part 6, Chapter 3, 2015. 